\title{
Simulation of the influence of dynamic loading on the stress- strain state of the natural and geoengineering environment
}

\author{
Natalya Remez ${ }^{1}$, Alina Dychko ${ }^{1, *}$, Vadym Bronytskyi ${ }^{1}$, Tetiana Hrebeniuk ${ }^{1}$, Rafael Bambirra Pereira ${ }^{2}$, and Petr Ekel $^{2}$ \\ ${ }^{1}$ Igor Sikorsky Kyiv Polytechnic Institute, Geoengineering Department, 115 Borshagivska Str., 03056, Kyiv, Ukraine \\ ${ }^{2}$ ASOTECH, Advanced System Optimization Technologies Ltd., 290 Prudente de Morais Ave., Santo Antônio, Belo Horizonte, MG, \\ 30380-002, Brazil
}

\begin{abstract}
The paper provides numerical simulation of the influence of dynamic loading on the stressstrain state of the natural and geoengineering technogenic environment taking into account the soil basis for forecasting its use as the basis of the structure. Paper demonstrates the impact of static and dynamic loading on the subsidence of the landfill. To take into account the liquid phase of the waste and the viscoplastic medium, Darcy's law is used as an equation of balance of forces. The body of the landfill is modeled by weak soil taking into account the creep, using the Soft Soil Creep model. The covering and underlying soil layers are described by the Coulomb - Mohr model. An effective method for calculating the sedimentation of natural and geoengineering environment on the example of a solid waste landfill, based on numerical modeling of the stress-strain state of the landfill and underlying soil using finite elements is developed. It is demonstrated that the largest subsidence is experienced by the landfill with sand, as the base soil, but in percentage terms the amount of subsidence with the maximum load relative to the initial subsidence without loading is the largest in clay $(33.7 \%)$. The obtained results must be taken into account when using landfills as a basis for buildings, structures, routes, recreational areas, etc.
\end{abstract}

\section{Introduction}

Due to the continuous growth of industry and, as a result, the increase in industrial and household waste, dumps and tailings of the mining industry, the area allocated for them is growing accordingly. For example, currently in Ukraine only landfills for solid waste occupy 160 thousand hectares of land.

Therefore, the question of the sustainability of such natural-technogenic geoengineering areas for their possible use in the future as a basis for engineering buildings and structures. As a result, scientists are faced with the task of assessing and predicting stability under static and dynamic loads [1].

Analysis of the literature demonstrated that experimental methods for assessing the resilience of natural-technogenic environments are costly and are not always effective. It is most expedient to apply mathematical modeling to take into account the stressstrain state of such medium, taking into account the underlying soil arrays and dynamic loads.

Mathematical models used to predict subsidence can be divided into rheological models, empirical models, models based on soil mechanics and models that take into account biodegradation.

Although properties of hard domestic wastes differ from properties of traditional soils, Kockel and Jessberger set that the landfills can be modelled by weak soil $[2,3]$. Sowers and Edil were the first, who applied principles of ordinary mechanics of soils for the estimation of sinking as a result of primary compression [4].

Marquez developed a composite rheological model to take into account the primary and secondary mechanisms of compression [6].

Park and Lee proposed a sedimentation model that takes into account time-dependent biodegradation of waste [7].

A common feature of mathematical models $[8,9]$ is that they take into account only waste, their behavior and properties, neglecting such an important component of the landfill as the soil underlying it. Type, strength, deformability, geotechnical properties of the underlying soil determine the stability of the natural and geoengineering environment, because it feels the greatest load. Currently, this issue is not studied.

The aim of the research is to substantiate theoretically the stability of the natural and geoengineering environment as the basis of structures, taking into account the stress-strain state of the underlying soils and dynamic loads to predict the stability of the landfill as the basis of structures.

To achieve this goal, the following research objectives are formulated:

- to research the impact of static and dynamic loading on the subsidence of the landfill using the developed methodology for selecting and predicting the

\footnotetext{
*Corresponding author: aodi@ukr.net
} 
possible use of the landfill as a basis for construction after its closure;

- to study the influence of the underlying soil and its stratification on the deformation of the landfill body using the developed mathematical model and methods of calculating the stress-strain state.

Sediment simulation taking into account the dynamic load predicts the suitability of a particular closed landfill for use as a road, highway, expressway, etc.

\section{Materials and methods}

Numerical modeling of the stress-strain state of the solid waste landfill and the soil base is carried out to predict the possibility of its use as the basis of a building or structure. To take into account the liquid phase of the waste and the visco-plastic medium, Darcy's law is used as an equation of balance of forces:

$$
-\nabla P-\frac{\eta}{K} \vec{u}+\rho \vec{f}=0,
$$

where $P$ - external pressure; $\rho$ - density; $\eta$ - dynamic viscosity; $g$ - acceleration of free fall; $\vec{u}$ - filtration rate; $\vec{f}$ - field of external forces; $K=\eta k / \rho g$ - the permeability coefficient, which characterizes the ability of a porous medium to pass fluid.

The complete system of equations of filtration of incompressible liquid includes the equation of incompressibility:

$$
\operatorname{div} \vec{u}=0 \text {, }
$$

and the continuity equation:

$$
\operatorname{div} \rho \vec{u}=-\rho m,
$$

where $m$ - the porosity of the soil.

It is assumed that the compressibility of the skeleton and pore fluid is small, which leads to a linear dependence of soil porosity on pressure.

The covering and underlying soil layers are described by the Coulomb - Mohr model, in which the complete yield condition consists of six flow surfaces and six plastic potential functions [10]:

$$
\begin{gathered}
f_{1 a}=\frac{1}{2}\left(\sigma_{2}^{\prime}-\sigma_{3}^{\prime}\right)+\frac{1}{2}\left(\sigma_{2}^{\prime}+\sigma_{3}^{\prime}\right) \sin \varphi- \\
-c \cos \varphi \leq 0 ; \\
f_{1 b}=\frac{1}{2}\left(\sigma_{3}^{\prime}-\sigma_{2}^{\prime}\right)+\frac{1}{2}\left(\sigma_{3}^{\prime}+\sigma_{2}^{\prime}\right) \sin \varphi- \\
-c \cos \varphi \leq 0 ; \\
f_{2 a}=\frac{1}{2}\left(\sigma_{3}^{\prime}-\sigma_{1}^{\prime}\right)+\frac{1}{2}\left(\sigma_{3}^{\prime}+\sigma_{1}^{\prime}\right) \sin \varphi- \\
-c \cos \varphi \leq 0 ; \\
f_{2 b}=\frac{1}{2}\left(\sigma_{1}^{\prime}-\sigma_{3}^{\prime}\right)+\frac{1}{2}\left(\sigma_{1}^{\prime}+\sigma_{3}^{\prime}\right) \sin \varphi- \\
-c \cos \varphi \leq 0 ; \\
f_{3 a}=\frac{1}{2}\left(\sigma_{1}^{\prime}-\sigma_{2}^{\prime}\right)+\frac{1}{2}\left(\sigma_{1}^{\prime}+\sigma_{2}^{\prime}\right) \sin \varphi- \\
-c \cos \varphi \leq 0 ;
\end{gathered}
$$

$$
\begin{gathered}
f_{3 b}=\frac{1}{2}\left(\sigma_{2}^{\prime}-\sigma_{1}^{\prime}\right)+\frac{1}{2}\left(\sigma_{2}^{\prime}+\sigma_{1}^{\prime}\right) \sin \varphi- \\
-c \cos \varphi \leq 0
\end{gathered}
$$

where $\sigma_{1}^{\prime}, \sigma_{2}^{\prime}, \sigma_{3}^{\prime}$ - normal stresses.

The condition $f_{j}=0$ for all surfaces of fluidity together shows a hexagonal cone in space of main tensions.

In addition to the surfaces of fluidity, the model of Coulomb - Mohr is presented by six plastic potential functions $\mathrm{g}$ :

$$
\begin{aligned}
& g_{1 a}=\frac{1}{2}\left(\sigma_{2}^{\prime}-\sigma_{3}^{\prime}\right)+\frac{1}{2}\left(\sigma_{2}^{\prime}+\sigma_{3}^{\prime}\right) \sin \psi ; \\
& g_{1 b}=\frac{1}{2}\left(\sigma_{3}^{\prime}-\sigma_{2}^{\prime}\right)+\frac{1}{2}\left(\sigma_{3}^{\prime}+\sigma_{2}^{\prime}\right) \sin \psi ; \\
& g_{2 a}=\frac{1}{2}\left(\sigma_{3}^{\prime}-\sigma_{1}^{\prime}\right)+\frac{1}{2}\left(\sigma_{3}^{\prime}+\sigma_{1}^{\prime}\right) \sin \psi ; \\
& g_{2 b}=\frac{1}{2}\left(\sigma_{1}^{\prime}-\sigma_{3}^{\prime}\right)+\frac{1}{2}\left(\sigma_{1}^{\prime}+\sigma_{3}^{\prime}\right) \sin \psi ; \\
& g_{3 a}=\frac{1}{2}\left(\sigma_{1}^{\prime}-\sigma_{2}^{\prime}\right)+\frac{1}{2}\left(\sigma_{1}^{\prime}+\sigma_{2}^{\prime}\right) \sin \psi ; \\
& g_{3 b}=\frac{1}{2}\left(\sigma_{2}^{\prime}-\sigma_{1}^{\prime}\right)+\frac{1}{2}\left(\sigma_{2}^{\prime}+\sigma_{1}^{\prime}\right) \sin \psi .
\end{aligned}
$$

The corner of dilatancy $\psi$ is needed for the design of positive plastic volume to deformations, that are in dense soils. The corner of internal friction $\varphi$ is used for the design of effective friction in soil in combination with the effective coupling of $c$.

The body of the landfill is modeled by weak soil taking into account the creep, using the model Soft Soil Creep (SSC) [11-12].

Advantage of this model before other is that she takes into account not only a primary compression but also creep and secondary compression, that is especially important for the grounds of hard domestic wastes, in that a secondary compression plays a major role. Its duration can present 10-30 years. It must be taken into account on the stage of planning of building of construction on the basis of ground. The feature of body of ground is him high compressibility. The large primary sinking of foundations and embankments, as a rule, is accompanied by the substantial sinking as a result of creep in later years. In such cases it is desirable to estimate a creep by means of eventual elements method.

Basic descriptions of model of SSC are: dependency inflexibility; a difference is between the primary loading and unloading-overload; secondary, dependency upon time compression; taking into account of preconsolidation tension; criteria of destruction of Coulomb-Mohr. The feature of design of ground is linear dependence of his tension on odometric inflexibility.

The total volume deformation $\varepsilon_{v}$, caused by the increase of effective stresses from the initial value $p_{0}$ ' to $p^{\prime}$ for the period of time $t_{c}+t^{\prime}$, consists of elastic $\varepsilon_{v}^{e}$ and viscoplastic $\varepsilon_{v}^{v p}$ components. The viscoplastic component is the sum of the deformation during the 
consolidation $\varepsilon_{v}^{v p}$ and after the consolidation $\varepsilon_{\mathrm{v}}^{\mathrm{vp}}$ ac The relationship between deformations is expressed as follows:

$$
\begin{gathered}
\varepsilon_{\mathrm{v}}=\varepsilon_{\mathrm{v}}^{\mathrm{e}}+\varepsilon_{\mathrm{v}_{\mathrm{c}}}^{\mathrm{vp}}+\varepsilon_{\mathrm{v}}^{\mathrm{vp}}{ }_{\mathrm{ac}} ; \\
\varepsilon_{v}^{e}=\kappa^{*} \ln \left(\frac{p^{\prime}}{p_{0}^{\prime}}\right) ; \\
\varepsilon_{v}^{v p}=\left(\lambda^{*}-\kappa^{*}\right) \ln \left(\frac{p_{p c}^{\prime}}{p_{0}^{\prime}}\right) ; \\
\varepsilon_{v a c}^{v p}=\mu^{*} \ln \left(\frac{\tau_{c}+t^{\prime}}{\tau_{c}}\right),
\end{gathered}
$$

where $\mu^{*}$ - modified creep coefficient; $\tau_{c}$ - consolidation time, which depends on the geometry of the sample under study; $t$ ' - elapsed time since the start of landfill loading; $\kappa^{*}-$ modified swelling coefficient; $\lambda^{*}-$ modified compression ratio (compression); $t_{c}$ - time of completion of the initial consolidation; $p$ ' - initial effective voltage; $p^{\prime}$ - effective voltage; $p_{p c}^{\prime}$ - effective pre-consolidation voltage.

Izotropic effective tension of $p^{\prime}$ is determined after a formula:

$$
p^{\prime}=\frac{1}{3}\left(\sigma_{x x}^{\prime}+\sigma_{y y}^{\prime}+\sigma_{z z}^{\prime}\right) .
$$

Attitude of model parameters following toward the internationally rationed parameters

$$
\begin{gathered}
\mu^{*}=\frac{C_{\alpha}}{2,3\left(1+e_{0}\right)} ; \quad \lambda^{*}=\frac{C_{c}}{2,3\left(1+e_{0}\right)} ; \\
\kappa^{*}=\frac{2 C_{s}}{2,3\left(1+e_{0}\right)},
\end{gathered}
$$

where $C_{c}$ - coefficient of compression, $C_{s}-$ swelling coefficient, $C_{\alpha}-$ coefficient of creep, $e_{0}-$ initial coefficient of porosity.

The equations for determining the free surface of the filtration flow when calculating the unstable filtration in the body of the landfill, taking into account the infiltration, must satisfy the following boundary conditions: 1) the pressure is equal to atmospheric; 2) the normal velocity component on the surface is missing. At the boundaries between the landfill layers and the soil base, the conditions of equality of normal stress components and filtration rate occur.

The initial conditions are: the filtration rate in the layers of the landfill and the underlying soil is zero; atmospheric pressure acts on the surface, each layer of waste and soil has its own initial physical and mechanical properties, the initial geometric parameters of the landfill are set.

\section{The results of studies of the stress- strain state of the natural-technogenic environment under dynamic loads}

The finite element method is used to solve the problem numerically. The calculation area is divided into 5200 triangular elements.
The landfill for which the simulation is performed consists of ten layers of waste, the thickness of each layer is $3 \mathrm{~m}$. Sedimentation is determined taking into account the step-by-step load of the landfill for 30 years after its closure.

Sand, loam and clay are considered as underlying soils, the physical and mechanical parameters of which are given in table 1.

The physical and mechanical parameters of wastes are given in table 2 .

Table 1. Physical-mechanical parameters of soils.

\begin{tabular}{|c|c|c|c|}
\hline \multirow{2}{*}{ Parameter } & \multicolumn{3}{|c|}{ Soil } \\
\cline { 2 - 4 } & Sand & Loam & Clay \\
\hline Deformation module, $E_{\text {ref }}$ & 18000 & 10000 & 9000 \\
\hline Poisson's ratio, $v$ & 0,34 & 0,34 & 0,34 \\
\hline Specific gravity of soil, $\gamma_{\text {unsat }}$ & 18,0 & 13 & 19,0 \\
\hline The proportion of water-saturated soil, $\gamma_{\text {sat }}$ & 20,7 & 14,6 & 21,8 \\
\hline $\begin{array}{c}\text { The filtration coefficient in the horizontal } \\
\text { direction, } k_{x}\end{array}$ & 0,5 & 0,006 & 0,004 \\
\hline $\begin{array}{c}\text { The filtration coefficient in the vertical } \\
\text { direction, } k_{y}\end{array}$ & 0,5 & 0,006 & 0,004 \\
\hline Coupling, $c$ & 2 & 13 & 17 \\
\hline The angle of internal friction, $\varphi$ & 31 & 14 & 13 \\
\hline
\end{tabular}

Table 2. Physical-mechanical parameters of wastes.

\begin{tabular}{|l|c|}
\hline \multicolumn{1}{|c|}{ Parameter } & Size \\
\hline Specific gravity $\gamma_{\text {unsat }},\left(\mathrm{kN} / \mathrm{m}^{3}\right)$ & 7,504 \\
\hline Specific gravity of water saturated soil $\gamma_{\text {sat }},\left(\mathrm{kN} / \mathrm{m}^{3}\right)$ & 10,0 \\
\hline Specific coupling $c,(\mathrm{kPa})$ & 25 \\
\hline Corner of internal friction $\varphi,($ degree$)$ & 20 \\
\hline Initial coefficient of porosity, $e_{0}$, (unit) & 0,4268 \\
\hline Coefficient of compression, $C_{C^{\prime}}$ (unit) & 0,3987 \\
\hline Coefficient of swelling, $C_{S}$, (unit) & 0,0394 \\
\hline
\end{tabular}

In [9], the sedimentation of the landfill without load is calculated. For the landfill with clay base, it is $3.83 \mathrm{~m}$, with loam $-4.47 \mathrm{~m}$, with sand $-4.95 \mathrm{~m}$. These data are used to determine the stress-strain state of the landfill 30 years after its closure and predict the possibility of its use as the basis of the road surface.

As a result of numerical calculation it is established that if the value of the vertical load is $50 \mathrm{kPa}$, it can be observed that deformations are achieved equal to $4.19 \mathrm{~m}$ for clay (Fig. 1) and 5.06 for sand (Fig. 2).

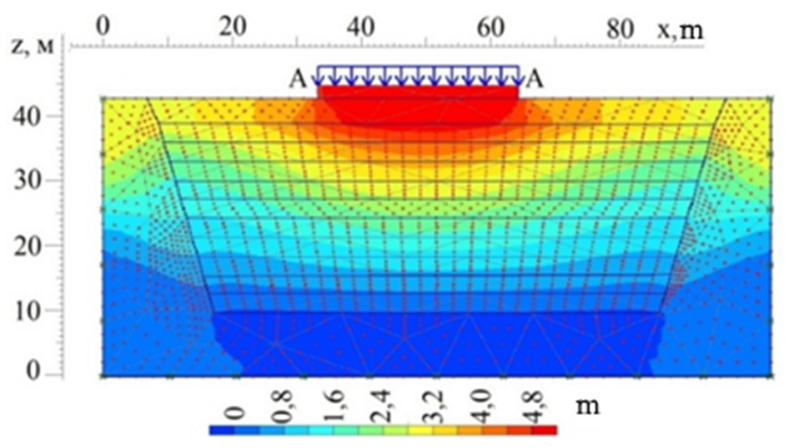

Fig. 1. Vertical deformations of the landfill with a vertical distributed dynamic load of $50 \mathrm{kPa}$ (clay at the base). 
With an increase in the load up to $100 \mathrm{kPa}$, the sediment of the landfill with a sand base increases by $4.2 \%$ (5.27 m) (Fig. 3), with clay - by 4.8\% (4.39 m) (Fig. 4).

It is established that at dynamic loads the underlying soils have a similar effect on the subsidence of the landfill, but the amount of subsidence is less than $0.5-4 \%$ depending on the underlying soil.

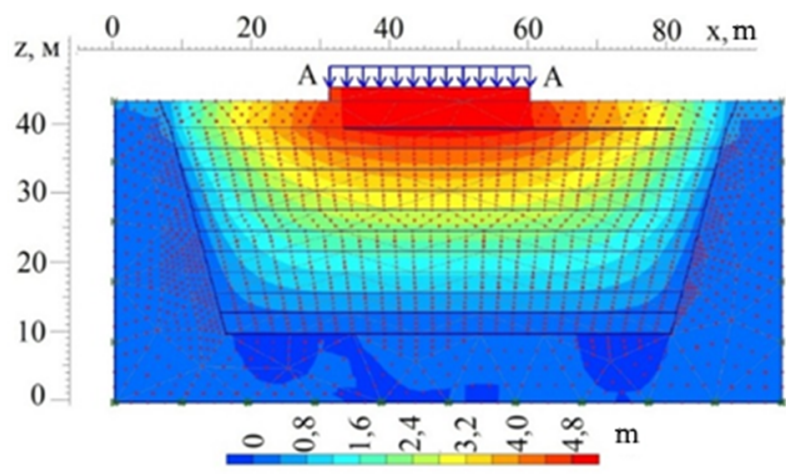

Fig. 2. Vertical deformations of the landfill with a vertical distributed dynamic load of $50 \mathrm{kPa}$ (sand at the base).

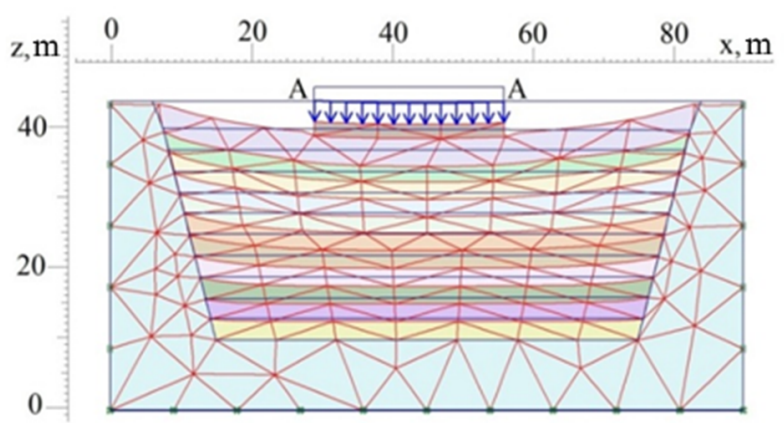

Fig. 3. Deformed calculation area of the landfill with a vertical distributed dynamic load of $100 \mathrm{kPa}$ (sand at the base).

The landfill with sand as the base soil undergoes the greatest vertical deformations. The amount of sedimentation with the maximum load in relation to the subsidence without load is the largest in clay (28.7\%), the minimum - in sand $(18.6 \%)$. It is established that with increasing load from 5 to $180 \mathrm{kPa}$, the sedimentation increases by $1.2-17.4 \%$ for sand and by $2.5-21 \%$ for clay.

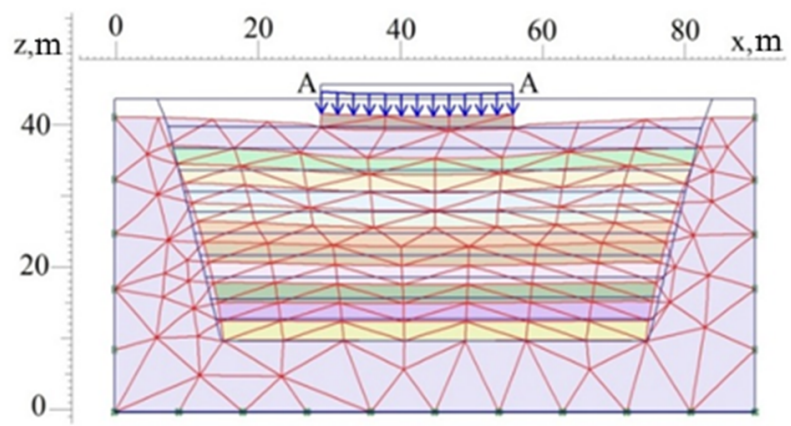

Fig. 4. Deformed calculation area of the landfill with a vertical distributed dynamic load of $100 \mathrm{kPa}$ (clay in the base).
Analytical dependences of subsidence of the landfill with different underlying soils on the value of static loads (Fig. 5) are obtained:

for sand

$$
y=0,0243 x^{2}+0,0686 x+3,79,
$$

for loam

$$
y=0,0043 x^{2}+0,2129 x+4,29,
$$

for clay

$$
y=0,0277 x^{2}-0,008 x+4,945
$$

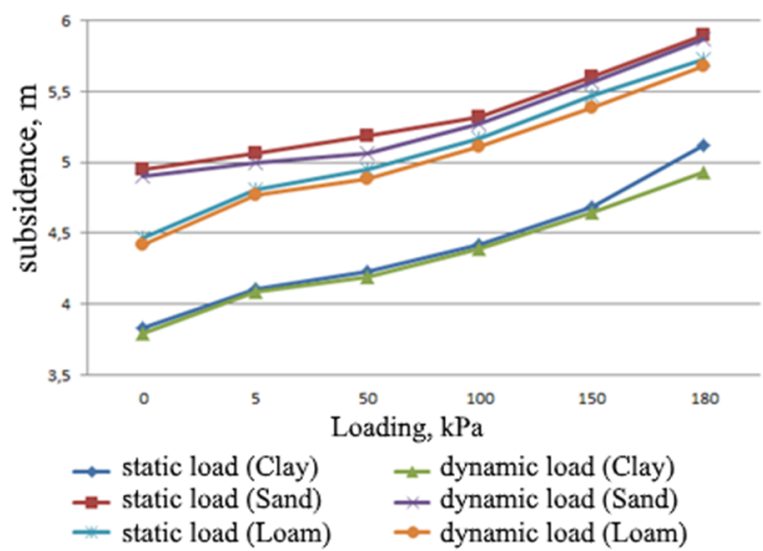

Fig. 5. Dependence of landfill subsidence with clay and sandy base soil on vertically distributed static [10] and dynamic loads.

Analytical dependences of subsidence of the landfill with different underlying soils on the value of dynamic loads (Fig. 5) are obtained:

for sand

$$
y=0,035 x^{2}-0,0516 x+4,928
$$

for loam

$$
y=0,0068 x^{2}+0,1922 x+4,266
$$

for clay

$$
y=0,0096 x^{2}+0,1491 x+3,672 .
$$

These dependences make it possible to predict the subsidence of landfills in order to assess their stability under static and dynamic loads of different nature: roads, highways and more.

For practical application of the worked out methodology the calculation of sinking was implemented on the example of landfill, located in Boryspil, Kyiv region.

A landfill was put into an operation in 2003 in accordance with a project in place of exhaust sand-pit, its area folds 6,5 hectare. The landfill's capacity of solid household waste was about 85 thousand tons per year. The total amount of waste is estimated in 0.723 million tons, the average depth is $18-20 \mathrm{~m}$. 
Sediment is determined in landfill site $(50 \times 50 \mathrm{~m})$, taking into account the step-by-step loading of the landfill with waste during 30 years after its closure, as approximately $90 \%$ of sediment occurs within the first five years and may continue for 25-30 years at a slower rate.

Clay and sand are in different parts of the landfill at its base. Modeling is performed for two options: with intermediate sand pouring every $3 \mathrm{~m}$ of waste and without it.

The calculated deformed area of the landfill with a clay underlying layer is presented in Fig. 6 and vertical deformations of the landfill with a sandy underlying layer is presented in Fig. 7.

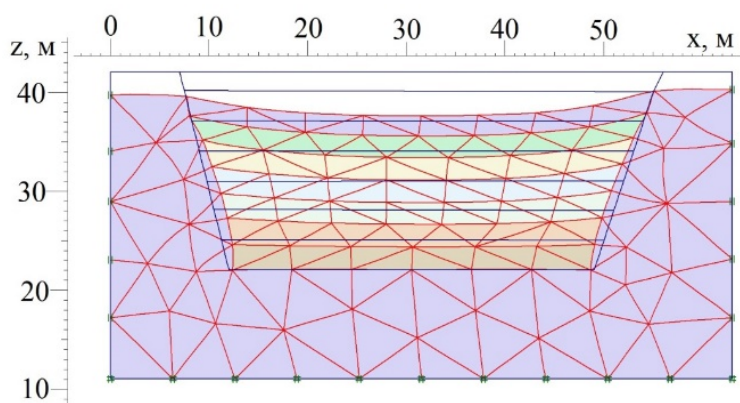

Fig. 6. Deformed calculation area of the landfill with a clay underlying layer.

From the analysis of numerical calculations it follows that the maximum vertical deformation at the clay base of the landfill is $2.27 \mathrm{~m}$. In case of sand as the underlying layer, the deformation increases significantly up to $2.61 \mathrm{~m}$.

It is found that at the landfill with overflow there are much smaller deformations compared to the landfill without overflow: $32 \%$ less at the landfill with a clay base, $30 \%$ less at the landfill with sand. Thus, if the underlying layer is clay (Fig. 8), then vertical deformations in $1.55 \mathrm{~m}$ are achieved, and in case of sand as the base (Fig. 9), - it is $1.82 \mathrm{~m}$. The percentage of sediment to the height of the landfill is $14.2 \%$ and $15.26 \%$ respectively.

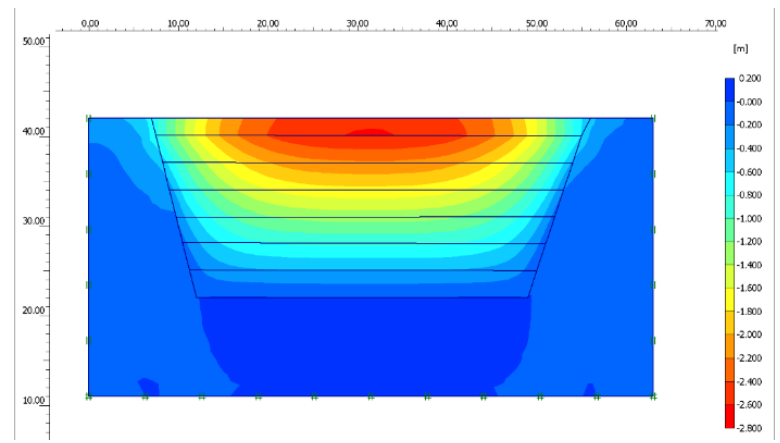

Fig. 7. Vertical deformations of the landfill with a sandy underlying layer.

It is established that at the same parameters of the landfill body during pouring, the landfill with a sand base is subject to greater sediment than the landfill with a clay base $(13 \%)$. There is a significant effect of overflow on the amount of deformation. Thus, for the clay base the sediment increases by $32 \%$, for the sand base - by $30 \%$.

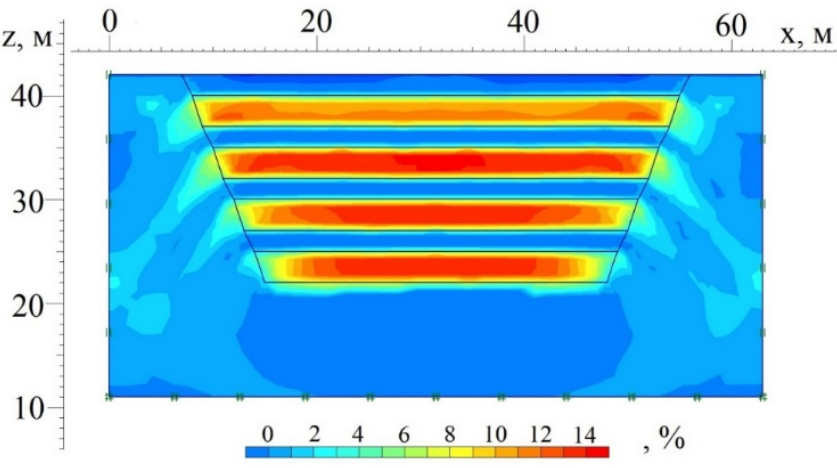

Fig. 8. Vertical displacements of the landfill with overflow and with a clay underlying layer (in percents).

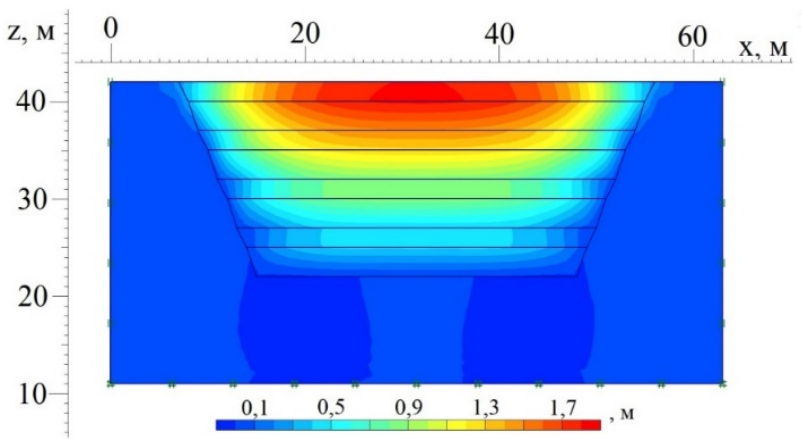

Fig. 9. Vertical deformations of the landfill with overflow and with a sandy underlying layer.

\section{Discussion of the results of the research}

As a result of numerical calculation, it is established that if the value of the vertical load is $50 \mathrm{kPa}$, it can be observed that deformations are achieved equal to $4.19 \mathrm{~m}$ for clay (Fig. 1) and 5.06 for sand (Fig. 2).

With an increase in the load up from 50 to $100 \mathrm{kPa}$, the sediment of the landfill with a sand base increases by $4.2 \%(5.27 \mathrm{~m})$ (Fig. 3), with clay - by $4.8 \%$ (4.39 m) (Fig. 4).

It is established that at dynamic loads the underlying soils have a similar effect on the subsidence of the landfill, but the amount of subsidence is less than $0.5-4 \%$ depending on the underlying soil.

As it follows from Figure 5 the landfill with sand as the base soil is subjected to the greatest vertical deformations. However, in percentage, the amount of sedimentation with the maximum load in relation to the initial subsidence without load is the largest in clay and is $33.7 \%$, the same figure in the sand $-19.2 \%$.

It is established that in the landfill with overflow there are much smaller deformations compared to the landfill without overflow: $32 \%$ less in the landfill with a clay base, $30 \%$ less in the landfill with sand. Thus, if the underlying layer is clay (Fig. 8), then vertical deformations of $1.55 \mathrm{~m}$ are achieved, and if the base is 
sand (Fig. 9) - $1.82 \mathrm{~m}$. The percentage of sediment to the height of the landfill is $14.2 \%$ and $15.26 \%$ respectively.

This is due to the fact that the more porous unstable soil of the sand base has reached the maximum subsidence in 30 years and is subsequently deformed to a lesser extent than the clay, which has not yet reached the final deformation. This established fact must be taken into account when designing and operating buildings for various purposes, built on closed landfills.

\section{Conclusions}

An effective method for calculating the sedimentation of natural and geoengineering environment on the example of a solid waste landfill, based on numerical modeling of the stress-strain state of the landfill and underlying soil using SSC models for the landfill and Coulomb-Mora for the soil base using finite elements is developed.

For the first time it is proposed to take into account the underlying soil when calculating the stability of the landfill, as it is one of the main factors in the formation of subsidence.

It is demonstrated that the largest subsidence is experienced by the landfill with sand, as the base soil, but in percentage terms the amount of subsidence with the maximum load relative to the initial subsidence without loading is the largest in clay $(33.7 \%)$.

It is established that in the landfill with overflow there are much smaller deformations compared to the landfill without overflow: $32 \%$ less at the landfill with a clay base, $30 \%$ less at the landfill with sand.

The obtained results must be taken into account when using landfills as a basis for buildings, structures, routes, recreational areas, etc.

As a result of the research it can be concluded that in the preliminary planning of the landfill it is necessary to provide for the overlapping of landfill layers with soils to choose areas with denser underlying soil to be able to use it after closure as a foundation. If there has been no prior planning and / or the basis is weak soil, it is necessary to use different methods of compaction or use it with less load (parks, recreational areas, golf courses).

\section{References}

1. G.L. Sivakumar Babu, K.R. Reddy, S.K. Chouskey, H.S. Kulkarni. Practice Periodical of Hazardous, Toxic and Radioactive Waste Management 14, 2, (2010). doi:10.1061/(ASCE)HZ.19448376.0000024

2. R. Kockel, H.L. Jessberger, in Proceedings of 11th European Conference for Soil Mechanics and Foundation Engineering, 2 (Danish Geotechnical Society, Copenhagen, Denmark, 1995)

3. M. Manassero, W.F. Van Impe, A. Bouazza, in Proceedings of 2nd International Congress on Environmental Geotechnics. Rotterdam, A.A. Balkema, 3 (1996)

4. T. B. Edil, V. J. Ranguette, W. W. Wuellner. ASTM Special Technical Publication 1070 (1990)
5. G.F. Sowers, in Proc. 8th Int. Conf. on Soil Mechanics and Foundation Engineering, Moscow, Russia, 2 (1973)

6. A.C.M Marques and O.M. Vilar. J. Geotech. Geoenviron. Eng. 129, 4 (2003)

7. H.I. Park, S.R. Lee. J. Resour. Manag. Technol. 24, 4 (1997)

8. R.E. Gibson, K.Y. Lo. Acta Polytech. Scand. 10 (1961)

9. F. Behnam, Fly Ash and Quicklime (University of Technology, Sydney, 2013)

10. P.A. Vermeer, H.P. Neher, in Proc. Int. Symp. "Beyond 2000 in Computational Geotechnics", Amsterdam, Balkema, Rotterdam (1999)

11. D. Rangeard, R. Zentar, N-E. Abriak, in Proceedings of Int. Conf. on Numer. Models in Geomech., NUMOG IX (2004)

12. W.T. Koiter, Progress in Solid Mechanics. NorthHolland, Amsterdam, 1, (1960)

13. N. Remez, A. Dychko, S. Kraychuk, N. Ostapchuk, L. Yevtieieva, V. Bronitskiy. LatJP 55, 3 (2018). doi:10.2478/lpts-2018-0018 